\title{
Synchronization stability for recurrent neural networks with time-varying delays
}

\author{
Ahmadjan Muhammadhaji*, Azhar Halik \\ College of Mathematics and Systems Science, Xinjiang University, Urumqi 830046 China
}

*Corresponding author, e-mail: ahmatjanam@aliyun.com

Received 8 Jul 2018

Accepted 9 Apr 2019

\begin{abstract}
This paper studies the general decay synchronization (GDS) of a class of recurrent neural networks (RNNs) with general activation functions and time-varying delays. By constructing suitable Lyapunov-Krasovskii functionals and employing useful inequality techniques, some sufficient conditions on the GDS of considered RNNs are established via a type of nonlinear control. In addition, an example with numerical simulations is presented to illustrate the obtained theoretical results.
\end{abstract}

KEYWORDS: general decay synchronization, general activation functions

MSC2010: 34D06 93B52

\section{INTRODUCTION}

Neural network dynamical systems have become one of the hot topics in modern applied mathematics. Its dynamic types of behaviour often include asymptotic stability, robust stability, local or global stability, synchronization stability, exponential stability, the existence of periodic solution, and polynomial stability ${ }^{1-10}$. As a mature and widely-accepted network system, recurrent neural network system has become one of the most important topic both in theory and applications, such as classification of image processing, pattern recognition, signal processing, associative memories, optimization problem ${ }^{1-16}$.

However, the time delays inevitably exist in natural and man-made systems and cannot be neglected. We can see from the results that the time delays have a great destabilizing influence on the implementation of neural networks ${ }^{1-20}$. There has been a lot of literature related to the study of recurrent neural networks with time delays ${ }^{6-16}$.

It is worth noting that the synchronization problem in neural network systems is one of the most basic and important concerns when we investigate the dynamical types of behaviour of recurrent neural networks (RNNs). Furthermore, the synchronization play an extremely important role in many fields of science including biology, climatology, sociology, ecology ${ }^{13-21}$. In view of the significance of the synchronization for delayed recurrent cellular neural networks (RCNNs), there are many important works have been developed to stabilize or synchronize neural networks and nonlinear systems ${ }^{13-24}$.

It is well known that the estimate of the convergent rate of synchronization is very interesting and useful for studying the synchronization of chaotic systems. In some cases, the convergence rates of the synchronization are not shown or very difficult to estimate. For example, consider the equation ${ }^{21}$ $\dot{y}(x)=-y^{3} / 2, x \geqslant 0$. Although the equation is asymptotically stable, it is very difficult to estimate the convergent rate of the solution.

However, researchers have recently investigated the synchronization problem for classes of chaotic neural networks (NNs) with continuous activations by introducing a new concept of synchronization, called general decay synchronization (GDS) ${ }^{21-24}$. This leads us to consider a new type of convergence rate, such as convergence with general decay. Furthermore, studies on the general decay synchronization for RNNs with time-varying delays are fairly rare. Hence based on the above analysis and reasons, we consider in this study the following $n$-dimensional RNNs with time-varying delays

$$
\begin{aligned}
\dot{x}_{i}(t)= & -c_{i} x_{i}(t)+\sum_{j=1}^{n} a_{i j} f_{j}\left(x_{j}(t)\right) \\
& +\sum_{j=1}^{n} b_{i j} g_{j}\left(x_{j}\left(t-\tau_{i j}(t)\right)\right) \\
& +\sum_{j=1}^{n} d_{i j} h_{j}\left(x_{j}\left(t-\sigma_{i j}(t)\right)\right)+I_{i},
\end{aligned}
$$


where $i \in \Upsilon \triangleq\{1,2, \ldots, n\}, n \geqslant 2$ denotes the number of neurons in the neural networks; $x_{i}(t)$ corresponds to the state variable of the $i$ th unit at time $t ; c_{i}>0$ denotes the rate with which the $i$ th neuron resets its potential to the resting state when isolated from the other neurons and inputs; $a_{i j}, b_{i j}$, and $d_{i j}$ are the connection weights between the $i$ th and $j$ th neurons at time $t ; f_{i}(\cdot), g_{i}(\cdot)$, and $h_{i}(\cdot)$ are the nonlinear activation functions; $I_{i}$ is the external input vector; and $\tau_{i j}(t)$ and $\sigma_{i j}(t)$ are the transmission time-varying delays satisfying $0 \leqslant$ $\tau_{i j}(t) \leqslant \tau_{i j}$ and $0 \leqslant \sigma_{i j}(t) \leqslant \sigma_{i j}$, respectively.

The main purpose of the study is to construct suitable Lyapunov-Krasovskii functionals and apply a method to establish some new sufficient conditions on the general decay synchronization ${ }^{22,23}$ for the system (1).

\section{PRELIMINARIES}

In this study, we use $\Upsilon=\{1,2, \ldots, n\}$ and $\mathbb{R}_{0}^{+}=$ $[0, \infty)$, unless otherwise stated. The initial conditions associated with the system (1) are given by

$$
x_{i}(s)=\varphi_{i}(s), \quad s \in[-\tau, 0], \quad i=1,2, \ldots, n
$$

where $\tau=\max _{i, j \in \Upsilon}\left\{\tau_{i j}, \sigma_{i j}\right\}$ and $\varphi(s)=$ $\left(\varphi_{1}(s), \varphi_{2}(s), \ldots, \varphi_{n}(s)\right) \in C([-\tau, 0], \mathbb{R}), \quad$ the Banach space of all continuous functions with norm

$$
\|\varphi\|=\sum_{i=1}^{n} \sup _{s \in[-\tau, 0]}\left|\varphi_{i}(s)\right| .
$$

Let $v=\left(v_{1}, v_{1}, \ldots, v_{n}\right) \in \mathbb{R}^{n}$ with norm

$$
\|v\|=\left(\sum_{i=1}^{n}\left|v_{i}\right|^{2}\right)^{1 / 2} \quad \text { or } \quad\|\mathrm{v}\|=\max _{\mathrm{i} \in \Upsilon}\left|\mathrm{v}_{\mathrm{i}}\right| .
$$

Throughout this paper, we assume that the following assumptions are satisfied.

$\left(\mathbf{H}_{1}\right)$ For each $j \in \Upsilon$, the activation functions $f_{j}(u), g_{j}(u), h_{j}(u)$ are continuous and there exist constants $L_{j}, H_{j}, K_{j}, N_{j}, M_{j}, O_{j}>0$ such that for all $v_{1}, v_{2} \in \mathbb{R}$,

$$
\begin{gathered}
\left|f_{j}\left(v_{1}\right)-f_{j}\left(v_{2}\right)\right| \leqslant L_{j}\left|v_{1}-v_{2}\right|+N_{j}, \\
\left|g_{j}\left(v_{1}\right)-g_{j}\left(v_{2}\right)\right| \leqslant H_{j}\left|v_{1}-v_{2}\right|+M_{j}, \\
\left|h_{j}\left(v_{1}\right)-h_{j}\left(v_{2}\right)\right| \leqslant K_{j}\left|v_{1}-v_{2}\right|+O_{j} .
\end{gathered}
$$

$\left(\mathbf{H}_{2}\right)$ Time-varying delays $\tau_{i j}(t)$ and $\sigma_{i j}(t)$ are differentiable and there exist real numbers $0 \leqslant \zeta_{i j}, \gamma_{i j}<1$ such that for any $t \geqslant 0$,

$$
0 \leqslant \dot{\tau}_{i j}(t) \leqslant \zeta_{i j} \text { and } 0 \leqslant \dot{\sigma}_{\mathrm{ij}}(\mathrm{t}) \leqslant \gamma_{\mathrm{ij}} .
$$

In this paper, we consider the system (1) as the driven system, and the response system is given as

$$
\begin{aligned}
\dot{y}_{i}(t)= & -c_{i} y_{i}(t)+\sum_{j=1}^{n} a_{i j} f_{j}\left(y_{j}(t)\right) \\
& +\sum_{j=1}^{n} b_{i j} g_{j}\left(y_{j}\left(t-\tau_{i j}(t)\right)\right) \\
& +\sum_{j=1}^{n} d_{i j} h_{j}\left(y_{j}\left(t-\sigma_{i j}(t)\right)\right)+I_{i}+u_{i}(t),
\end{aligned}
$$

where $u_{i}(t)$ is the controller to be designed.

Let $e_{i}(t)=y_{i}(t)-x_{i}(t)$. Then from (1) and (2), the error of the dynamical system is

$$
\begin{aligned}
\dot{e}_{i}(t)= & -c_{i} e_{i}(t)+\sum_{j=1}^{n} a_{i j} \tilde{f}_{j}+\sum_{j=1}^{n} b_{i j} \tilde{g}_{i j} \\
& +\sum_{j=1}^{n} d_{i j} \tilde{h}_{i j}+u_{i}(t),
\end{aligned}
$$

where

$$
\begin{aligned}
& \tilde{f}_{j}=f_{j}\left(y_{j}(t)\right)-f_{j}\left(x_{j}(t)\right), \\
& \tilde{g}_{i j}=g_{j}\left(y_{j}\left(t-\tau_{i j}(t)\right)\right)-g_{j}\left(x_{j}\left(t-\tau_{i j}(t)\right)\right), \\
& \tilde{h}_{i j}=h_{j}\left(y_{j}\left(t-\sigma_{i j}(t)\right)\right)-h_{j}\left(x_{j}\left(t-\sigma_{i j}(t)\right)\right) .
\end{aligned}
$$

We now give the definitions of $\psi$-type functions and GDS.

Definition 1 [Refs. 22, 23]. A function $\psi: \mathbb{R}_{0}^{+} \rightarrow$ $[1, \infty)$ is said to be $\psi$-type function if it satisfies the following conditions:

(i) It is differentiable and nondecreasing;

(ii) $\psi(0)=1$ and $\psi(\infty)=\infty$;

(iii) $\tilde{\psi}(t)=\dot{\psi}(t) / \psi(t)$ is nondecreasing and $\psi^{*}=\sup _{t \geqslant 0} \tilde{\psi}(t)<\infty$, where $\dot{\psi}(t)$ is the time derivative of $\psi(t)$;

(iv) for any $t, s \geqslant 0, \psi(t+s) \leqslant \psi(t) \psi(s)$.

It is not difficult to check that functions $\psi(t)=\mathrm{e}^{\alpha t}$ and $\psi(t)=(1+t)^{\alpha}$ for any $\alpha>0$ satisfy the above four conditions, thus are $\psi$-type functions.

Definition 2 [Refs. 22, 23]. The drive-response systems (1) and (2) are said to be general decay synchronized if there exists a constant $\varepsilon>0$ and a $\psi$-type function $\psi$ such that for any solutions $x(t)=\left(x_{1}(t), x_{2}(t), \ldots, x_{n}(t)\right)$ and $y(t)=$ $\left(y_{1}(t), y_{2}(t), \ldots, y_{n}(t)\right)$ of the systems (1) and (2), respectively,

$$
\limsup _{t \rightarrow \infty} \frac{\log \|y(t)-x(t)\|}{\log \psi(t)} \leqslant \varepsilon,
$$


where $\varepsilon$ is the convergence rate when synchronization error approaches zero.

$\left(\mathbf{H}_{3}\right)$ For the functions $\psi(t), \tilde{\psi}(t)$ given in Definition 1 , there exist a function $\varrho(t) \in C\left(\mathbb{R}, \mathbb{R}_{0}^{+}\right)$ and a constant $\delta$ such that for any $t \geqslant 0$

$$
\tilde{\psi}(t) \leqslant 1, \quad \sup _{t \in[0, \infty)} \int_{0}^{t} \psi^{\delta}(s) \varrho(s) \mathrm{d} s<\infty .
$$

We now present a useful lemma which is essential to this study.

Lemma 1 (Refs. 22, 23) . Under the assumption $\left(\mathbf{H}_{3}\right)$, assume that the synchronization error $e(t)=$ $y(t)-x(t)$ of the driver-response systems (1) and (2) satisfy the differential equation $\dot{e}(t)=g\left(t, e_{t}\right)$, where $e_{t}=e(t+s)$ for $s \in[-\tau, 0]$ and the function $g\left(t, e_{t}\right)$ is locally bounded. If there exists a differentiable functional $V\left(t, e_{t}\right): \mathbb{R}_{0}^{+} \times C \rightarrow \mathbb{R}_{0}^{+}$and positive constants $\lambda_{1}, \lambda_{2}$ such that for any $\left(t, e_{t}\right) \in \mathbb{R}_{0}^{+} \times C$

$$
\begin{aligned}
\left(\lambda_{1}\|e(t)\|\right)^{2} & \leqslant V\left(t, e_{t}\right) \\
\left.\frac{\mathrm{d} V}{\mathrm{~d} t}\left(t, e_{t}\right)\right|_{(3)} & \leqslant-\delta V\left(t, e_{t}\right)+\lambda_{2} \varrho(t),
\end{aligned}
$$

where $x(t)$ and $y(t)$ are solutions of the systems (1) and (2), respectively, and $\delta>0$ and $\varrho(t)$ are defined in $\left(\mathbf{H}_{3}\right)$. Then the driver-response systems (1) and (2) are general decay synchronized in the sense of Definition 2, and the convergence rate is $\delta / 2$.

\section{MAIN RESULTS}

In this section, we will obtain some sufficient conditions to insure the GDS of the systems (1) and (2). First letting $C_{i j}$ and $D_{i j}$ be numbers greater than zero, and under assumption $\left(\mathbf{H}_{3}\right)$ designing the controller $u_{i}(t)$ of the response system (2) as follows.

$$
u_{i}(t)=-\alpha_{i} \operatorname{sgn}\left(e_{i}(t)\right)-\frac{\beta_{i}\|e(t)\|^{2} e_{i}(t)}{e_{i}^{2}(t)+\varrho(t)},
$$

where $\beta_{i}$ and $\alpha_{i}$ are control gains satisfying

$$
\begin{gathered}
-c_{i}-\beta_{i}+\sum_{j=1}^{n}\left(\frac{A_{j i}}{1-\zeta_{j i}}+\frac{B_{j i}}{1-\gamma_{j i}}+\tau_{j i} C_{j i}+\sigma_{j i} D_{j i}\right. \\
\left.+\frac{\left|a_{i j}\right| L_{j}+\left|a_{j i}\right| L_{i}+\left|b_{i j}\right| H_{j}+\left|d_{i j}\right| K_{j}}{2}\right)<0, \\
-\alpha_{i}+\sum_{j=1}^{n}\left(\left|a_{i j}\right| N_{j}+\left|b_{i j}\right| M_{j}+\left|d_{i j}\right| O_{j}\right)<0,
\end{gathered}
$$

where $A_{i j}=\left|b_{i j}\right| H_{j} / 2$ and $B_{i j}=\left|d_{i j}\right| K_{j} / 2, i, j \in \Upsilon$.

Based on the nonlinear controller (6), the following theorem is obtained.
Theorem 1 Suppose $\left(\mathbf{H}_{1}\right)-\left(\mathbf{H}_{3}\right)$ hold. Then the response network (2) is general decay synchronized with the drive network (1) under the nonlinear controller (6) if the control gains $\beta_{i}$ satisfy (7).

Proof: Firstly, we construct the following LyapunovKrasovskii functional.

$$
\begin{aligned}
V_{1}(t)=\sum_{i=1}^{n} \frac{1}{2} e_{i}^{2}(t) & +\sum_{i=1}^{n} \sum_{j=1}^{n} \int_{t-\tau_{i j}(t)}^{t} \frac{A_{i j} e_{j}^{2}(s)}{1-\zeta_{i j}} \mathrm{~d} s \\
& +\sum_{i=1}^{n} \sum_{j=1}^{n} \int_{t-\sigma_{i j}(t)}^{t} \frac{B_{i j} e_{j}^{2}(s)}{1-\gamma_{i j}} \mathrm{~d} s .
\end{aligned}
$$

Calculating the derivative of $V_{1}(t)$ along the trajectory of the system (3), we obtain

$$
\begin{aligned}
& \dot{V}_{1}(t)=\sum_{i=1}^{n}\left\{e _ { i } ( t ) \left[-c_{i} e_{i}(t)+\sum_{j=1}^{n} a_{i j} \tilde{f}_{j}+\sum_{j=1}^{n} b_{i j} \tilde{g}_{i j}\right.\right. \\
& \left.\left.+\sum_{j=1}^{n} d_{i j} \tilde{h}_{i j}-\alpha_{i} \operatorname{sgn}\left(e_{i}(t)\right)-\frac{\left.\beta_{i}\|e(t)\|^{2} e_{i}(t)\right]}{e_{i}^{2}(t)+\varrho(t)}\right]\right\} \\
& +\sum_{i=1}^{n} \sum_{j=1}^{n} A_{i j}\left[\frac{e_{j}^{2}(t)}{1-\zeta_{i j}}-\frac{1-\dot{\tau}_{i j}(t)}{1-\zeta_{i j}} e_{j}^{2}\left(t-\tau_{i j}(t)\right)\right] \\
& +\sum_{i=1}^{n} \sum_{j=1}^{n} B_{i j}\left[\frac{e_{j}^{2}(t)}{1-\gamma_{i j}}-\frac{1-\dot{\sigma}_{i j}(t)}{1-\gamma_{i j}} e_{j}^{2}\left(t-\sigma_{i j}(t)\right)\right] \\
& \leqslant \sum_{i=1}^{n}\left[-c_{i} e_{i}^{2}(t)+\sum_{j=1}^{n}\left|a_{i j} e_{i}(t) \tilde{f}_{j}\right|+\sum_{j=1}^{n}\left|b_{i j} e_{i}(t) \tilde{g}_{i j}\right|\right. \\
& \left.+\sum_{j=1}^{n}\left|d_{i j} e_{i}(t) \tilde{h}_{i j}\right|-\alpha_{i}\left\|e_{i}(t)\right\|-\frac{\beta_{i}\|e(t)\|^{2} e_{i}^{2}(t)}{e_{i}^{2}(t)+\varrho(t)}\right] \\
& +\sum_{i=1}^{n} \sum_{j=1}^{n}\left(\frac{A_{i j}}{1-\zeta_{i j}}+\frac{B_{i j}}{1-\gamma_{i j}}\right) e_{j}^{2}(t) \\
& -\sum_{i=1}^{n} \sum_{j=1}^{n} A_{i j} e_{j}^{2}\left(t-\tau_{i j}(t)\right)-\sum_{i=1}^{n} \sum_{j=1}^{n} B_{i j} e_{j}^{2}\left(t-\sigma_{i j}(t)\right) .
\end{aligned}
$$

Using $\left(\mathbf{H}_{1}\right)$ and $a b \leqslant\left(a^{2}+b^{2}\right) / 2$, we have

$$
\begin{aligned}
& \sum_{i=1}^{n} \sum_{j=1}^{n}\left|a_{i j} e_{i}(t) \tilde{f}_{j}\right| \\
& \quad \leqslant \sum_{i=1}^{n} \sum_{j=1}^{n}\left|a_{i j} e_{i}(t)\right|\left(L_{j}\left|e_{j}(t)\right|+N_{j}\right) \\
& \quad \leqslant \sum_{i=1}^{n} \sum_{j=1}^{n}\left|a_{i j}\right|\left[\frac{L_{j}}{2}\left(e_{i}^{2}(t)+e_{j}^{2}(t)\right)+N_{j}\left|e_{i}(t)\right|\right] .
\end{aligned}
$$


Similarly, we have

$$
\begin{aligned}
& \sum_{i=1}^{n} \sum_{j=1}^{n}\left|b_{i j} e_{i}(t) \tilde{g}_{i j}\right| \\
& \leqslant \sum_{i=1}^{n} \sum_{j=1}^{n}\left|b_{i j} e_{i}(t)\right|\left(H_{j}\left|e_{j}\left(t-\tau_{i j}(t)\right)\right|+M_{j}\right) \\
& \leqslant \sum_{i=1}^{n} \sum_{j=1}^{n}\left|b_{i j}\right|\left[\frac{H_{j}}{2}\left(e_{i}^{2}(t)+e_{j}^{2}\left(t-\tau_{i j}(t)\right)\right)+M_{j}\left|e_{i}(t)\right|\right]
\end{aligned}
$$

and

$$
\begin{aligned}
& \sum_{i=1}^{n} \sum_{j=1}^{n}\left|d_{i j} e_{i}(t) \tilde{h}_{i j}\right| \\
& \leqslant \sum_{i=1}^{n} \sum_{j=1}^{n}\left|d_{i j} e_{i}(t)\right|\left(K_{j}\left|e_{j}\left(t-\sigma_{i j}(t)\right)\right|+O_{j}\right) \\
& \leqslant \sum_{i=1}^{n} \sum_{j=1}^{n}\left|d_{i j}\right|\left[\frac{K_{j}}{2}\left(e_{i}^{2}(t)+e_{j}^{2}\left(t-\sigma_{i j}(t)\right)\right)+O_{j}\left|e_{i}(t)\right|\right] .
\end{aligned}
$$

We construct the Lyapunov-Krasovskii functional,

$$
\begin{aligned}
V_{2}(t)=\sum_{i=1}^{n} \sum_{j=1}^{n} \int_{-\tau_{i j}}^{0} \int_{t+s}^{t} C_{i j} e_{j}^{2}(\epsilon) \mathrm{d} \epsilon \mathrm{d} s \\
\\
\quad+\sum_{i=1}^{n} \sum_{j=1}^{n} \int_{-\sigma_{i j}}^{0} \int_{t+s}^{t} D_{i j} e_{j}^{2}(\epsilon) \mathrm{d} \epsilon \mathrm{d} s .
\end{aligned}
$$

Calculating the derivative of $V_{2}(t)$, we obtain

$$
\begin{aligned}
\dot{V}_{2}(t) & \leqslant \sum_{i=1}^{n} \sum_{j=1}^{n}\left(\tau_{i j} C_{i j}+\sigma_{i j} D_{i j}\right) e_{j}^{2}(t) \\
& -\sum_{i=1}^{n} \sum_{j=1}^{n}\left[C_{i j} \int_{t-\tau_{i j}}^{t} e_{j}^{2}(\epsilon) \mathrm{d} \epsilon+D_{i j} \int_{t-\sigma_{i j}}^{t} e_{j}^{2}(\epsilon) \mathrm{d} \epsilon\right] .
\end{aligned}
$$

Finally, we construct the following LyapunovKrasovskii functional, $V(t)=V_{1}(t)+V_{2}(t)$. Then, there exists a scalar $\chi>1$ such that

$$
\begin{aligned}
& \frac{1}{2} \sum_{i=1}^{n} e_{i}^{2}(t) \leqslant V(t), \\
& V(t) \leqslant \chi \sum_{i=1}^{n} e_{i}^{2}(t)+\frac{\chi}{E} \sum_{i=1}^{n} \sum_{j=1}^{n}\left[C_{i j} \int_{t-\tau_{i j}}^{t} e_{j}^{2}(\epsilon) \mathrm{d} \epsilon\right. \\
&\left.+D_{i j} \int_{t-\sigma_{i j}}^{t} e_{j}^{2}(\epsilon) \mathrm{d} \epsilon\right]
\end{aligned}
$$

where $E=\min _{i \in \Upsilon}\left\{E_{i}\right\}$ with

$$
\begin{array}{r}
E_{i}=c_{i}+\beta_{i}-\sum_{j=1}^{n}\left[\frac{A_{j i}}{1-\zeta_{j i}}+\frac{B_{j i}}{1-\gamma_{j i}}+\tau_{j i} C_{j i}+\sigma_{j i} D_{j i}\right. \\
\left.+\frac{\left|a_{i j}\right| L_{j}+\left|a_{j i}\right| L_{i}+\left|b_{i j}\right| H_{j}+\left|d_{i j}\right| K_{j}}{2}\right]>0 .
\end{array}
$$

Calculating the derivative of $V(t)$ and from above results, we obtain

$$
\begin{aligned}
& \dot{V}(t) \leqslant \sum_{i=1}^{n}\left[-c_{i}+\sum_{j=1}^{n}\left(\frac{A_{j i}}{1-\zeta_{j i}}+\frac{B_{j i}}{1-\gamma_{j i}}+\tau_{j i} C_{j i}\right.\right. \\
& \left.\left.+\sigma_{j i} D_{j i}+\frac{\left|a_{i j}\right| L_{j}+\left|a_{j i}\right| L_{i}+\left|b_{i j}\right| H_{j}+\left|d_{i j}\right| K_{j}}{2}\right)\right] e_{i}^{2}(t) \\
& -\sum_{i=1}^{n}\left[\alpha_{i}-\sum_{j=1}^{n}\left(\left|a_{i j}\right| N_{j}+\left|b_{i j}\right| M_{j}+\left|d_{i j}\right| K_{j}\right)\right]\left|e_{i}(t)\right| \\
& -\sum_{i=1}^{n} \frac{\beta_{i}\|e(t)\|^{2} e_{i}^{2}(t)}{e(t)^{2}+\varrho(t)}-\sum_{i=1}^{n} \sum_{j=1}^{n}\left(C_{i j} \int_{t-\tau_{i j}}^{t} e_{j}^{2}(\epsilon) \mathrm{d} \epsilon\right. \\
& \left.+D_{i j} \int_{t-\sigma_{i j}}^{t} e_{j}^{2}(\epsilon) \mathrm{d} \epsilon\right)-\sum_{i=1}^{n} \beta_{i} e_{i}^{2}(t)+\sum_{i=1}^{n} \beta_{i} e_{i}^{2}(t) \\
& \leqslant \sum_{i=1}^{n}\left[-c_{i}-\beta_{i}+\sum_{j=1}^{n}\left(\frac{A_{j i}}{1-\zeta_{j i}}+\frac{B_{j i}}{1-\gamma_{j i}}+\tau_{j i} C_{j i}\right.\right. \\
& \left.\left.+\sigma_{j i} D_{j i}+\frac{\left|a_{i j}\right| L_{j}+\left|a_{j i}\right| L_{i}+\left|b_{i j}\right| H_{j}+\left|d_{i j}\right| K_{j}}{2}\right)\right] e_{i}^{2}(t) \\
& +\sum_{i=1}^{n} \beta_{i} e_{i}^{2}(t)-\sum_{i=1}^{n} \frac{\beta_{i}\|e(t)\|^{2} e_{i}^{2}(t)}{\|e(t)\|^{2}+\varrho(t)} \\
& -\sum_{i=1}^{n} \sum_{j=1}^{n}\left(C_{i j} \int_{t-\tau_{i j}}^{t} e_{j}^{2}(\epsilon) \mathrm{d} \epsilon+D_{i j} \int_{t-\sigma_{i j}}^{t} e_{j}^{2}(\epsilon) \mathrm{d} \epsilon\right) \\
& \leqslant \sum_{i=1}^{n}-E_{i} e_{i}^{2}(t)+\max _{i \in \Upsilon}\left\{\beta_{i}\right\} \frac{\|e(t)\|^{2} \varrho(t)}{\|e(t)\|^{2}+\varrho(t)} \\
& -\sum_{i=1}^{n} \sum_{j=1}^{n}\left(C_{i j} \int_{t-\tau_{i j}}^{t} e_{j}^{2}(\epsilon) \mathrm{d} \epsilon+D_{i j} \int_{t-\sigma_{i j}}^{t} e_{j}^{2}(\epsilon) \mathrm{d} \epsilon\right) .
\end{aligned}
$$

Since

$$
\frac{\|e(t)\|^{2} \varrho(t)}{\|e(t)\|^{2}+\varrho(t)} \leqslant \frac{\|e(t)\|^{2} \varrho(t)}{\|e(t)\|^{2}}=\varrho(t),
$$

we have

$$
\begin{aligned}
\dot{V}(t) & \leqslant \sum_{i=1}^{n}-E_{i} e_{i}^{2}(t)+\beta \varrho(t) \\
& -\sum_{i=1}^{n} \sum_{j=1}^{n}\left[C_{i j} \int_{t-\tau_{i j}}^{t} e_{j}^{2}(\epsilon) \mathrm{d} \epsilon+D_{i j} \int_{t-\sigma_{i j}}^{t} e_{j}^{2}(\epsilon) \mathrm{d} \epsilon\right],
\end{aligned}
$$


where $\beta=\max _{i \in \Upsilon}\left\{\beta_{i}\right\}>0$. Taking $\delta$ such that $\delta \chi<E$, (9) and (10) give

$$
\begin{gathered}
\dot{V}(t)+\delta V(t) \leqslant \sum_{i=1}^{n}-E_{i} e_{i}^{2}(t)+\beta \varrho(t) \\
-\sum_{i=1}^{n} \sum_{j=1}^{n}\left(C_{i j} \int_{t-\tau_{i j}}^{t} e_{j}^{2}(\epsilon) \mathrm{d} \epsilon+D_{i j} \int_{t-\sigma_{i j}}^{t} e_{j}^{2}(\epsilon) \mathrm{d} \epsilon\right) \\
+\delta\left[\chi \sum_{i=1}^{n} e_{i}^{2}(t)+\frac{\chi}{E} \sum_{i=1}^{n} \sum_{j=1}^{n}\left(C_{i j} \int_{t-\tau_{i j}}^{t} e_{j}^{2}(\epsilon) \mathrm{d} \epsilon\right.\right. \\
\left.\left.\quad+D_{i j} \int_{t-\sigma_{i j}}^{t} e_{j}^{2}(\epsilon) \mathrm{d} \epsilon\right)\right] \\
\leqslant(\delta \chi-E) \sum_{i=1}^{n} e_{i}^{2}(t)+\sum_{i=1}^{n} \sum_{j=1}^{n}\left(\frac{\delta \chi}{E}-1\right) \\
\times\left(C_{i j} \int_{t-\tau_{i j}}^{t} e_{j}^{2}(\epsilon) \mathrm{d} \epsilon+D_{i j} \int_{t-\sigma_{i j}}^{t} e_{j}^{2}(\epsilon) \mathrm{d} \epsilon\right)+\beta \varrho(t),
\end{gathered}
$$

which implies that

$$
\dot{V}(t)+\delta V(t) \leqslant \beta \varrho(t) .
$$

Then by Lemma 1, the drive-response systems (1) and (2) achieve GDS under the adaptive nonlinear controller (6). The convergence rate of $e(t)$ approaching zero is $\delta / 2$.

Remark 1 The function $\psi$ is used as the decay function, so $\psi$-type stability is also said to be stability with general decay rate. When $\psi(t)=e^{\alpha t}$ and $\psi(t)=(1+t)^{\alpha}$ for any $\alpha>0, \psi$-type stability may be specialized as exponential synchronization and polynomial synchronization.

In addition, the controller (6) in the system (2) becomes

$$
u_{i}(t)=-\frac{\beta_{i}\|e(t)\|^{2} e_{i}(t)}{e_{i}^{2}(t)+\varrho(t)}, \quad i \in \Upsilon .
$$

From Theorem 1, we have the following corollary.

Corollary 1 Suppose $\left(\mathbf{H}_{1}\right),\left(\mathbf{H}_{2}\right),\left(\mathbf{H}_{3}\right)$ hold. Then the response network (2) can be general decay synchronized with the drive network (1) under the nonlinear controller (12) if the control gains $\beta_{i}$ satisfy the inequality

$$
\begin{gathered}
-c_{i}-\beta_{i}+\sum_{j=1}^{n}\left[\frac{A_{j i}}{1-\zeta_{j i}}+\frac{B_{j i}}{1-\gamma_{j i}}+\tau_{j i} C_{j i}+\sigma_{j i} D_{j i}\right. \\
\left.+\frac{\left|a_{i j}\right| L_{j}+\left|a_{j i}\right| L_{i}+\left|b_{i j}\right| H_{j}+\left|d_{i j}\right| K_{j}}{2}\right]<0
\end{gathered}
$$

Remark 2 To achieve GDS of the considered master-slave systems, Wang et $\mathrm{al}^{22,23}$ used the controllers $u_{i}(t)$ as

$$
-G_{i} e(t)-G_{2} \operatorname{sgn}(e(t))-\frac{\|A\|_{\infty}^{2}\|e(t)\|^{2} e(t)}{2\left(\|A\|_{\infty}\|e(t)\|^{2}+\varrho(t)\right)},
$$

where $G_{1}=\operatorname{diag}\left(\epsilon_{1}, \epsilon_{2}, \ldots, \epsilon_{n}\right)$ and $G_{2}=$ $\operatorname{diag}\left(\varepsilon_{1}, \varepsilon_{2}, \ldots, \varepsilon_{n}\right)$. However, in this study we used the simpler and more efficient controller given in (6) and (12). Hence the results of this study is an improvement and extension of the results obtained in Refs. 22, 23.

If in $\left(\mathrm{H}_{1}\right)$ we assume that the activation functions $f_{j}(u), g_{j}(u), h_{j}(u)$ are globally Lipschitz, i.e., the constants $N_{j}=M_{j}=O_{j}=0$, the $\left(\mathbf{H}_{1}\right)$ turns to $\left(\overline{\mathbf{H}}_{1}\right)$.

$\left(\overline{\mathbf{H}}_{1}\right) f_{j}(u), g_{j}(u), h_{j}(u)$ are globally Lipschitz continuous, i.e., there exist constants $L_{j}, H_{j}, K_{j}>0$ such that for all $v_{1}, v_{2} \in \mathbb{R}$,

$$
\begin{aligned}
& \left|f_{j}\left(v_{1}\right)-f_{j}\left(v_{2}\right)\right| \leqslant L_{j}\left|v_{1}-v_{2}\right|, \\
& \left|g_{j}\left(v_{1}\right)-g_{j}\left(v_{2}\right)\right| \leqslant H_{j}\left|v_{1}-v_{2}\right|, \\
& \left|h_{j}\left(v_{1}\right)-h_{j}\left(v_{2}\right)\right| \leqslant K_{j}\left|v_{1}-v_{2}\right| .
\end{aligned}
$$

In the system (1), if $d_{i j}=0$, then it is reduced to the following $n$-dimensional RNNs with time-varying delays

$$
\begin{aligned}
\dot{x}_{i}(t)=-c_{i} x_{i}(t) & +\sum_{j=1}^{n} a_{i j} f_{j}\left(x_{j}(t)\right) \\
& +\sum_{j=1}^{n} b_{i j} g_{j}\left(x_{j}\left(t-\tau_{i j}(t)\right)\right)+I_{i} .
\end{aligned}
$$

Accordingly, the response system (2) becomes

$$
\begin{aligned}
\dot{y}_{i}(t)= & -c_{i} y_{i}(t)+\sum_{j=1}^{n} a_{i j} f_{j}\left(y_{j}(t)\right) \\
& +\sum_{j=1}^{n} b_{i j} g_{j}\left(y_{j}\left(t-\tau_{i j}(t)\right)\right)+I_{i}+u_{i}(t) .
\end{aligned}
$$

Accordingly, the assumptions $\left(\mathbf{H}_{1}\right)$ and $\left(\overline{\mathbf{H}}_{1}\right)$ turn to:

$\left(\mathbf{H}_{1}^{*}\right)$ For each $j \in \Upsilon$, the activation functions $f_{j}(u)$ and $g_{j}(u)$ are continuous and there exist constants $L_{j}, H_{j}, N_{j}, M_{j}>0$ such that for $v_{1}, v_{2} \in \mathbb{R}$,

$$
\begin{aligned}
& \left|f_{j}\left(v_{1}\right)-f_{j}\left(v_{2}\right)\right| \leqslant L_{j}\left|v_{1}-v_{2}\right|+N_{j}, \\
& \left|g_{j}\left(v_{1}\right)-g_{j}\left(v_{2}\right)\right| \leqslant M_{j}\left|v_{1}-v_{2}\right|+M_{j} .
\end{aligned}
$$


$\left(\overline{\mathbf{H}}_{1}^{*}\right)$ For each $j \in \Upsilon$, the activation functions $f_{j}(u), g_{j}(u)$ are globally Lipschitz continuous, i.e., there exist constants $L_{j}, H_{j}>0$ such that for $v_{1}, v_{2} \in \mathbb{R}$,

$$
\begin{aligned}
& \left|f_{j}\left(v_{1}\right)-f_{j}\left(v_{2}\right)\right| \leqslant L_{j}\left|v_{1}-v_{2}\right|, \\
& \left|g_{j}\left(v_{1}\right)-g_{j}\left(v_{2}\right)\right| \leqslant H_{j}\left|v_{1}-v_{2}\right| .
\end{aligned}
$$

From Theorem 1 we have the following corollaries.

Corollary 2 Suppose $\left(\mathbf{H}_{1}^{*}\right),\left(\mathbf{H}_{2}\right)$ and $\left(\mathbf{H}_{3}\right)$ hold. Then the response network (15) can be general decay synchronized with the drive network (14) under the nonlinear controller (6) if the control gains $\beta_{i}$ satisfy the inequalities

$$
\begin{gathered}
-c_{i}-\beta_{i}+\sum_{j=1}^{n}\left(\frac{A_{j i}}{1-\zeta_{j i}}\right. \\
\left.+\frac{\left|a_{i j}\right| L_{j}+\left|a_{j i}\right| L_{i}+\left|b_{i j}\right| H_{j}}{2}+\tau_{j i} C_{j i}\right)<0, \\
\quad-\alpha_{i}+\sum_{j=1}^{n}\left(\left|a_{i j}\right| N_{j}+\left|b_{i j}\right| M_{j}\right)<0 .
\end{gathered}
$$

Corollary 3 Suppose $\left(\overline{\mathbf{H}}_{1}^{*}\right),\left(\mathbf{H}_{2}\right)$ and $\left(\mathbf{H}_{3}\right)$ hold. Then the response network (15) can be general decay synchronized with the drive network (14) under the nonlinear controller (12) if the control gains $\beta_{i}$ satisfy the inequality

$$
\begin{aligned}
-c_{i}-\beta_{i} & +\sum_{j=1}^{n}\left(\frac{A_{j i}}{1-\zeta_{j i}}\right. \\
& \left.+\frac{\left|a_{i j}\right| L_{j}+\left|a_{j i}\right| L_{i}+\left|b_{i j}\right| H_{j}}{2}+\tau_{j i} C_{j i}\right)<0 .
\end{aligned}
$$

\section{NUMERICAL SIMULATIONS}

In this section, an example is given to illustrate the effectiveness of the obtained results.

Example 1 For $n=2$, we consider the following chaotic recurrent neural network system with timevarying delays

$$
\begin{aligned}
\dot{x}_{i}(t)= & -c_{i} x_{i}(t)+\sum_{j=1}^{2} a_{i j} f_{j}\left(x_{j}(t)\right) \\
& +\sum_{j=1}^{2} b_{i j} g_{j}\left(x_{j}\left(t-\tau_{i j}(t)\right)\right) \\
& +\sum_{j=1}^{2} d_{i j} h_{j}\left(x_{j}\left(t-\sigma_{i j}(t)\right)\right)+I_{i},
\end{aligned}
$$

where $f_{1}(u)=f_{2}(u)=\tanh (u), g_{1}(u)=g_{2}(u)=$ $\tanh (u)-0.1 \sinh (u)$, and $h_{1}(u)=h_{2}(u)=\tanh (u)+$ $0.1 \cosh (u)$. The parameters of the system (16) are $c_{1}=c_{2}=1, a_{11}=1.55, a_{12}=-0.1, a_{21}=-1, a_{22}=$ $0.4, b_{11}=-1.5, b_{12}=-0.6, b_{21}=0.5, b_{22}=-0.95$, $d_{11}=-0.9, d_{12}=-0.4, d_{21}=-0.5, d_{22}=-0.85$, $\tau_{i j}(t)=\mathrm{e}^{t} /\left(1+\mathrm{e}^{t}\right), \sigma_{i j}(t)=\mathrm{e}^{t} /\left(2+\mathrm{e}^{t}\right)$, and $I_{i}=0$ for $i=1,2$.

The numerical simulation of the system (16) with initial values $x_{1}(s)=0.4$ and $x_{2}(s)=0.5$ for $s \in$ $[-1,0]$ is represented in Fig. 1 . We can see that the system (16) has a chaotic attractor.

The corresponding response system is

$$
\begin{aligned}
& \dot{y}_{i}(t)=-c_{i} y_{i}(t)+\sum_{j=1}^{2} a_{i j} f_{j}\left(y_{j}(t)\right) \\
& \quad+\sum_{j=1}^{2} b_{i j} g_{j}\left(y_{j}\left(t-\tau_{i j}(t)\right)\right) \\
& \quad+\sum_{j=1}^{2} d_{i j} h_{j}\left(y_{j}\left(t-\sigma_{i j}(t)\right)\right)+I_{i}+u_{i}(t),
\end{aligned}
$$

where $c_{i}, a_{i j}, b_{i j}, d_{i j}, f_{j}(t), g_{j}(t), h_{j}(t), \tau_{i j}(t), \sigma_{i j}(t)$, and $I_{i}$ are the same as in the system (16), and the nonlinear controllers $u_{i}(t)$ are designed as

$$
u_{i}(t)=-\alpha_{i} \operatorname{sgn}\left(e_{i}(t)\right)-\frac{\beta_{i}\|e(t)\|^{2} e_{i}(t)}{e_{i}^{2}(t)+\varrho(t)},
$$

where $e_{i}(t)=y_{i}(t)-x_{i}(t)$ for $i=1,2$.

It is not difficult to estimate that $L_{j}=H_{j}=K_{j}=$ $1, N_{j}=0.01, M_{j}=0.02, O_{j}=0.015$, and $\tau_{i j}=\sigma_{i j}=$ 1. Thus the assumptions $\left(\mathbf{H}_{1}\right)$ and $\left(\mathbf{H}_{2}\right)$ are satisfied. Letting $\varrho(t)=\mathrm{e}^{-0.1 t}, \psi(t)=\mathrm{e}^{t}$ and choosing $\alpha_{1}=3.5, \alpha_{2}=3.2, \beta_{1}=4, \beta_{2}=3.7$. Then the assumption $\left(\mathrm{H}_{3}\right)$ and (7) of Theorem 1 are satisfied. Hence the drive-response systems (16) and (17) can achieve GDS under the controller (18). The time evolution of synchronization errors between the systems (16) and (17) are demonstrated in Fig. 2, where the initial values of the response system (17) are chosen to be $y_{1}(s)=0.5$ and $y_{2}(s)=0$ for $s \in$ $[-1,0]$. The synchronization curves between the systems (16) and (17) are shown in Fig. 3.

\section{CONCLUSIONS}

In this study, we investigate the GDS problem for a class of RNNs with general activation functions and time-varying delays. Some sufficient conditions on the general decay synchronization of the driveresponse systems (1) and (2) are obtained by constructing suitable Lyapunov-Krasovskii functionals 

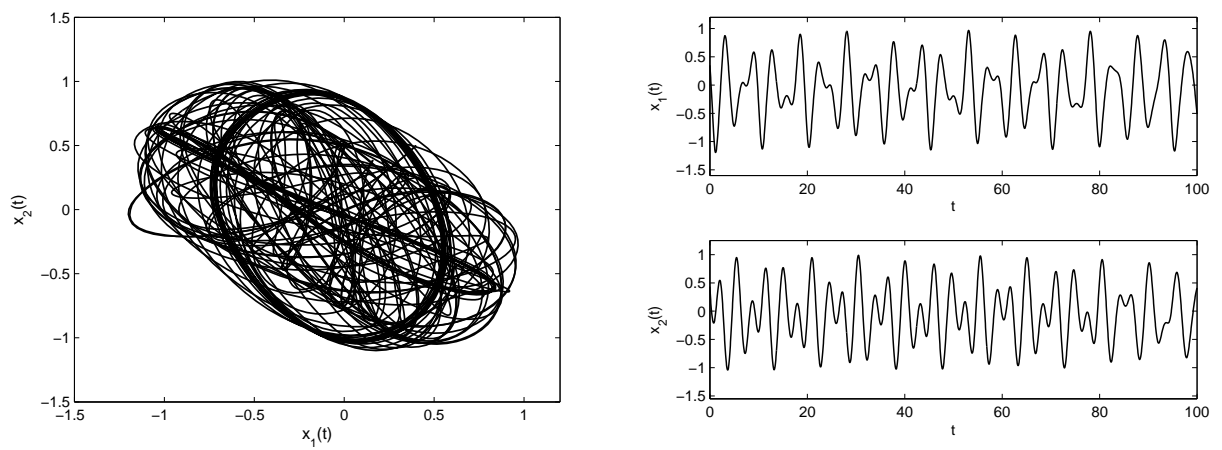

Fig. 1 The chaotic behaviour of delayed recurrent neural network system (16).
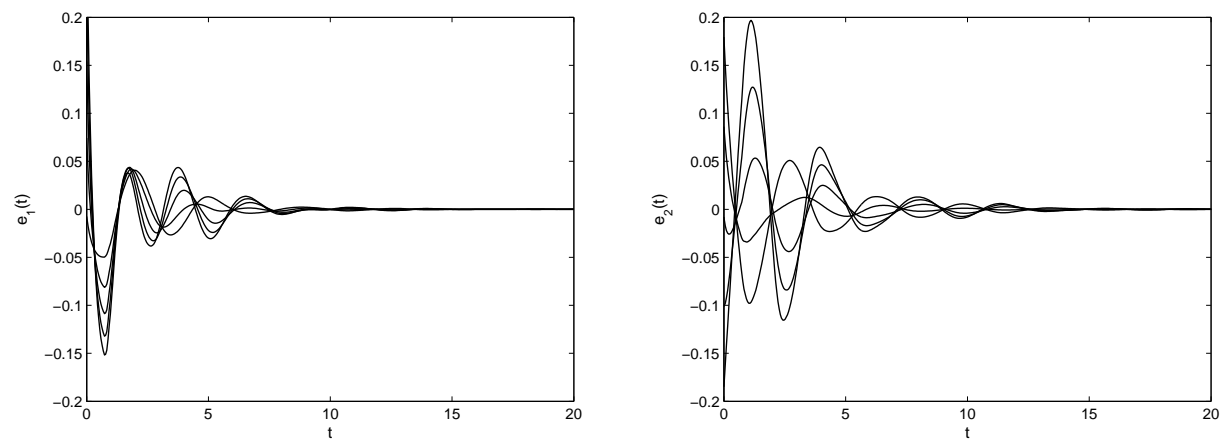

Fig. 2 The evaluation of synchronization error $e_{1}(t)$ and $e_{2}(t)$ in Example 1 .

and employing useful inequalities. In addition, an example and its numerical simulations are given to validate the theoretical results in this study. Furthermore, it is believed that our approaches and obtained results may bring some new guidance for the synchronization stability study of other type neural networks with delays such as, delayed RNNs with discontinuous activations ${ }^{25}$, delayed fuzzy cellular neural networks with discontinuous activations ${ }^{26,27}$ and some kinds of delayed complex-valued neural networks ${ }^{28-30}$. The GDS problem for the above mentioned neural networks with delays may be of interest to other researchers.

Acknowledgements: This work was supported by the National Natural Science Foundations of China (Grant Nos. 11601464 and 11662020).

\section{REFERENCES}

1. Zeng Z, Wang J, Liao X (2003) Global exponential

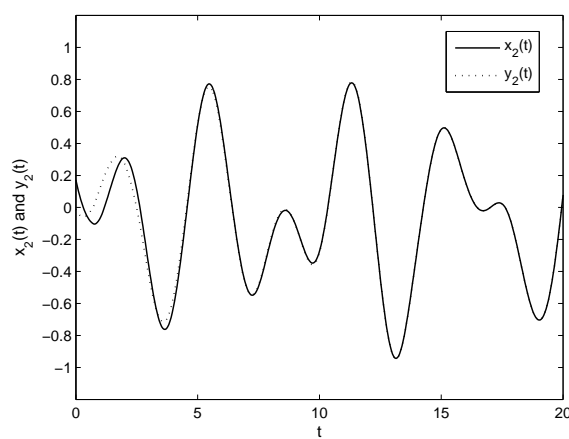

Fig. 3 Synchronization curves of $x_{1}(t), y_{1}(t)$ and $x_{2}(t), y_{2}(t)$ in Example 1 . 
stability of a general class of recurrent neural networks with time-varying delays. IEEE Trans Circuits Syst I 50, 1353-8.

2. Cao J, Wang J (2004) Absolute exponential stability of recurrent neural networks with Lipschitzcontinuous activation functions and time delays. Neural Netw 17, 379-90.

3. Chen B, Wang J (2005) Global exponential periodicity of a class of recurrent neural networks with oscillating parameters and time-varying delays. IEEE Trans Neural Netw 16, 1440-8.

4. Cao J, Wang J (2005) Global asymptotic and robust stability of recurrent neural networks with time delays. IEEE Trans Circuits Syst I 52, 417-26.

5. Li C, Liao X (2006) Robust stability and robust periodicity of delayed recurrent neural networks with noise disturbance. IEEE Trans Circuits Syst I 53, 2265-73.

6. Huang X, Cao J, Ho Daniel WC (2006) Existence and attractivity of almost periodic solution for recurrent neural networks with unbounded delays and variable coefficients. Nonlinear Dyn 45, 337-51.

7. Zhang H, Wang Z, Liu D (2008) Global asymptotic stability of recurrent neural networks with multiple time-varying delays. IEEE Trans Neural Netw 19, 855-73.

8. Lou X, Cui B (2008) Delay-dependent criteria for global robust periodicity of uncertain switched recurrent neural networks with time-varying delay. IEEE Trans Neural Netw 19, 549-57.

9. Hu J, Wang J (2012) Global stability of complexvalued recurrent neural networks with time-delays. IEEE Trans Neural Netw Learn Syst 23, 853-65.

10. Sun J, Chen J (2013) Stability analysis of static recurrent neural networks with interval time-varying delay. Appl Math Comput 221, 111-20.

11. Wen S, Zeng Z, Huang T, Chen Y (2013) Passivity analysis of memristor-based recurrent neural networks with time-varying delays. J Frankl Inst 350, 2354-70.

12. Zhou L, Zhang Y (2016) Global exponential periodicity and stability of recurrent neural networks with multi-proportional delays. ISA Trans 60, 89-95.

13. Wu A, Wen S, Zeng Z (2012) Synchronization control of a class of memristor-based recurrent neural networks. Inf Sci 183, 106-16.

14. Jiang M, Wang S, Mei J, Shen Y (2015) Finite-time synchronization control of a class of memristor-based recurrent neural networks. Neural Netw 63, 133-40.

15. Wu A, Zeng Z, Zhu X, Zhang J (2011) Exponential synchronization of memristor-based recurrent neural networks with time delays. Neurocomputing 74, 3043-50.

16. Li T, Fei S, Zhang K (2008) Synchronization control of recurrent neural networks with distributed delays. Physica A 387, 982-96.
17. Abdurahman A, Jiang H, Teng Z (2017) Lag synchronization for Cohen-Grossberg neural networks with mixed time-delays via periodically intermittent control. Int J Comput Math 94, 275-95.

18. Muhammadhaji A, Abdurahman A, Jiang H (2017) Finite-time synchronization of complex dynamical networks with time-varying delays and nonidentical nodes. J Ctrl Sci Eng 2017, 1-13.

19. Hu C, Jiang H, Teng Z (2010) Fuzzy impulsive control and synchronization of general chaotic system. Acta Appl Math 109, 463-85.

20. Hu M, Xu Z (2008) Adaptive feedback controller for projective synchronization. Nonlinear Anal RWA 9, 1253-60.

21. Sader M, Abdurahman A, Jiang H (2019) General decay lag synchronization for competitive neural networks with constant delays. Neural Process Lett (In press).

22. Wang L, Shen Y, Zhang G (2016) Synchronization of a class of switched neural networks with timevarying delays via nonlinear feedback control. IEEE Trans Cyber 46, 2300-310.

23. Wang L, Shen Y, Zhang G (2016) General decay synchronization stability for a class of delayed chaotic neural networks with discontinuous activations. Neurocomputing $179,169-75$.

24. Abdurahman A, Jiang $\mathrm{H}$, Hu C (2017) General decay synchronization of memristor-based CohenGrossberg neural networks with mixed time-delays and discontinuous activations. J Frankl Inst 354, 7028-52.

25. Duan L, Huang L, Fang X (2017) Finite-time synchronization for recurrent neural networks with discontinuous activations and time-varying delays. AIPChaos 27, 013101.

26. Duan L, Fang X, Fu Y (2019) Global exponential synchronization of delayed fuzzy cellular neural networks with discontinuous activations. Int $J$ Mach Learn Cyber 10, 579-89.

27. Duan L, Wei H, Huang L (2019) Finite-time synchronization of delayed fuzzy cellular neural networks with discontinuous activations. Fuzzy Set Syst 361, 56-70.

28. Song Q, Yan H, Zhao Z, Liu Y (2016) Global exponential stability of complex-valued neural networks with both time-varying delays and impulsive effects. Neural Netw 79, 108-16.

29. Song Q, Yan H, Zhao Z, Liu Y (2016) Global exponential stability of impulsive complex-valued neural networks with both asynchronous time-varying and continuously distributed delays. Neural Netw $\mathbf{8 1}$, $1-10$.

30. Song Q, Yan H, Zhao Z, Liu Y (2018) Boundedness and global robust stability analysis of delayed complex-valued neural networks with interval parameter uncertainties. Neural Netw 103, 55-62. 